\title{
Characterization, development and multiplexing of microsatellite markers in three commercially exploited reef fish and their application for stock identification
}

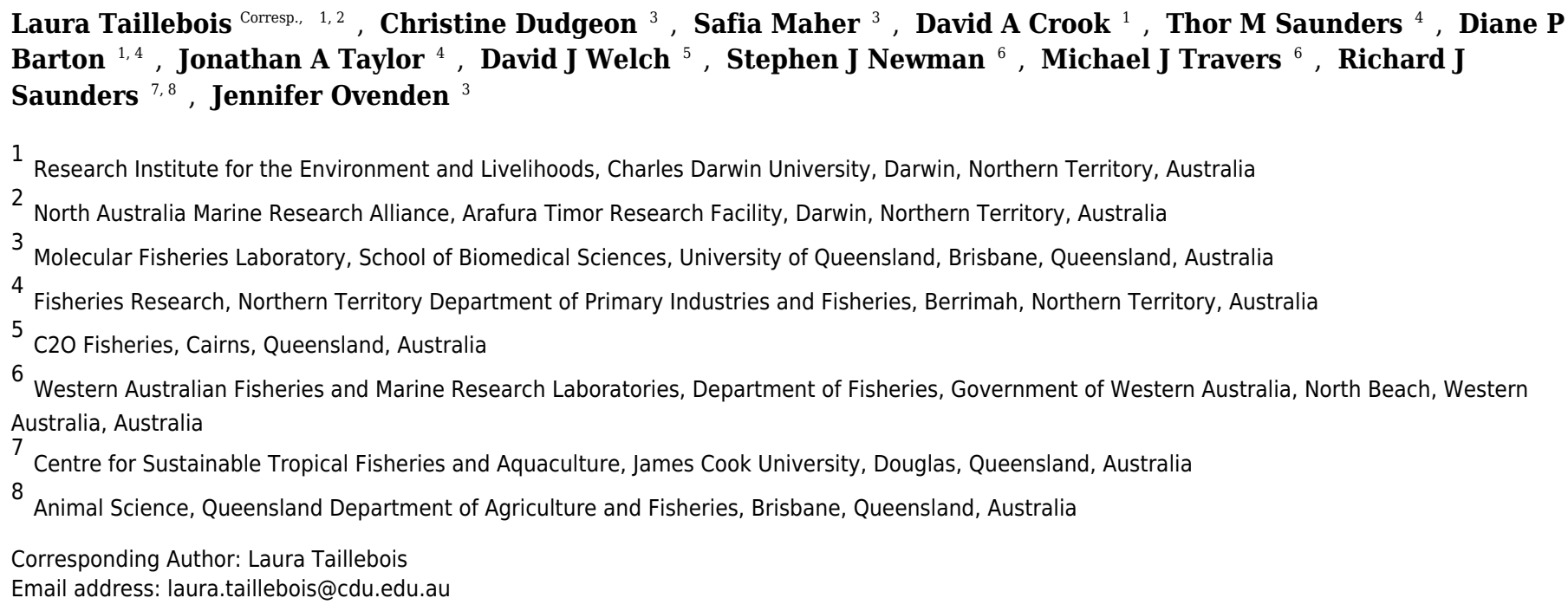

Thirty-four microsatellite loci were isolated from three reef fish species; golden snapper Lutjanusjohnii, black jewfish Protonibeadiacanthus and grass emperor Lethrinuslaticaudis using a next generation sequencing approach. Both lonTorrent single reads and Illumina MiSeq paired-end reads were used, with the latter demonstrating a higher quality of reads than the lonTorrent. From the 1-1.5 million raw reads per species, we successfully obtained 10 to 13 polymorphic loci for each species, which satisfied stringent design criteria. We developed multiplex panels for the amplification of the golden snapper and the black jewfish loci, as well as post-amplification pooling panels for the grass emperor loci. The microsatellites characterized in this work were tested across three locations of northern Australia. The microsatellites we developed can detect population differentiation across northern Australia and may be used for genetic structure studies and stock identification. 
1 Characterization, development and multiplexing of microsatellite markers in three 2 commercially exploited reef fish and their application for stock identification

3

4 Laura Taillebois $^{1,2}$, Christine Dudgeon ${ }^{3}$, Safia Maher ${ }^{3}$, David A. Crook ${ }^{1}$, Thor Saunders ${ }^{4}$, Diane 5 P. Barton ${ }^{1,4}$, Jonathan Taylor ${ }^{4}$, David J. Welch ${ }^{5}$, Stephen J. Newman ${ }^{6}$, Michael J. Travers ${ }^{6}$, 6 Richard J. Saunders ${ }^{7,8}$ \& Jennifer Ovenden ${ }^{3}$ 7

$8{ }^{1}$ Research Institute for the Environment and Livelihoods, Charles Darwin University, Darwin, 9 NT 0810, Australia

$10{ }^{2}$ North Australia Marine Research Alliance, Arafura Timor Research Facility, Darwin, NT 0810, 11 Australia

$12{ }^{3}$ Molecular Fisheries Laboratory, School of Biomedical Sciences, The University of Queensland, 13 St. Lucia, QLD 4072, Australia

144 Fisheries Research, Northern Territory Department of Primary Industries and Fisheries, 15 Berrimah, NT 0810, Australia

$16{ }^{5} \mathrm{C}_{2} \mathrm{O}$ Fisheries, Cairns, QLD 4870, Australia

176 Western Australian Fisheries and Marine Research Laboratories, Department of Fisheries, 18 Government of Western Australia, P.O. Box 20, North Beach, WA 6920, Australia

$19{ }^{7}$ Centre for Sustainable Tropical Fisheries and Aquaculture, James Cook University, Douglas, 20 QLD 4814, Australia

$21{ }^{8}$ Animal Science, Queensland Department of Agriculture and Fisheries, QLD, Australia 22

23 Corresponding auhtor: Laura Taillebois, Email: laura.taillebois@cdu.edu.au. 24 


\section{Abstract}

26

27 Thirty-four microsatellite loci were isolated from three reef fish species; golden snapper 28 Lutjanus johnii, black jewfish Protonibea diacanthus and grass emperor Lethrinus laticaudis 29 using a next generation sequencing approach. Both IonTorrent single reads and Illumina MiSeq 30 paired-end reads were used, with the latter demonstrating a higher quality of reads than the 31 IonTorrent. From the 1-1.5 million raw reads per species, we successfully obtained 10 to 13 32 polymorphic loci for each species, which satisfied stringent design criteria. We developed 33 multiplex panels for the amplification of the golden snapper and the black jewfish loci, as well as 34 post-amplification pooling panels for the grass emperor loci. The microsatellites characterized in 35 this work were tested across three locations of northern Australia. The microsatellites we 36 developed can detect population differentiation across northern Australia and may be used for 37 genetic structure studies and stock identification. 


\section{Introduction}

Microsatellites are hypervariable, nuclear-encoded and codominant-inherited markers used for a variety of aquaculture and fisheries applications, including determining the spatial extent of fisheries stocks and other important applications of population genetics. De novo discovery of microsatellites is required for analyses in the laboratory with each non-model species studied, however the costs are high and the procedure involving cloning is time-consuming (Peters et al. 2009). The alternative to de novo development is cross-species amplification where existing microsatellite loci of related species are used on the target species; but this is often hampered by the lack of conserved flanking sequences of microsatellites or the lack of data on related species. The adoption of Next-Generation Sequencing (NGS) by researchers using microsatellite loci has made the discovery of microsatellite markers easier (Gardner et al. 2011) and is becoming the preferred method for developing microsatellites (Abdelkrim et al. 2009; Castoe et al. 2010; Fernandez-Silva et al. 2013). Once the microsatellites are identified, major cost and time reductions in the laboratory are achieved through polymerase chain reaction (PCR) multiplexing. The challenge of PCR multiplexing is to combine several microsatellite primers into one PCR cocktail to amplify several microsatellite loci at the same time.

Herein, we describe the discovery, characterization, development and multiplexing of microsatellite loci of three reef fish species of commercial and recreational significance: golden snapper (Lutjanus johnii, Lutjanidae), black jewfish (Protonibea diacanthus, Sciaenidae) and grass emperor (Lethrinus laticaudis, Lethrinidae). Lutjanus johnii, is a highly prized sport and food fish and is harvested in the commercial, recreational, charter and indigenous sectors of northern Australia and many other fisheries worldwide (Allen, Swainston \& Ruse 1997). The catch of L.johnii has been declining in the Northern Territory since 1997 and this species is considered overfished (Grubert et al. 2013; Saunders et al. 2014a). Sciaenids form the basis of commercial and recreational fisheries of tropical and temperate regions worldwide (Lenanton \& Potter 1987; Rutherford et al. 1989) and several large species are considered threatened or vulnerable due to over-fishing (Rao et al. 1992; Saunders et al. 2014b; True, Loera \& Castro 1997). Among Sciaenid species, P. diacanthus is vulnerable to over-exploitation because of its predictable aggregating behavior (Bowtell 1995; Bowtell 1998; Phelan 2001). Lethrinus laticaudis is considered an excellent eating fish and is targeted by commercial fishers 
70 and recreational anglers across northern Australia (Coleman 2004). Although L. laticaudis is

71 considered robust to fishing pressure (Grubert, Kuhl \& Penn 2010) due to its high reproductive 72 capacity (i.e. serial batch spawners, high spawning frequency, high batch fecundity) (Ayvazian, 73 Chatfield \& Keay 2004), it is heavily exploited in some areas. These three fish species are of 74 high economic value and the sustainability of the fisheries they support is potentially threatened 75 by over harvesting and thus requires the development of suitable management programs. The 76 development of genetic tools is necessary to further investigate their population genetics and 77 assess stock structure.

78 In this study, we provide novel polymorphic microsatellite loci for the three species. We 79 also describe a fast and cost-effective protocol for species-specific microsatellite marker 80 discovery using genomic sequencing and multiplexing. Finally, we explore the relevance of the 81 described microsatellite markers for further population genetics by looking at the genetic 82 differences found between two locations in the Northern Territory for the three study species. 83 This will inform us on the potential to use these markers for the identification of stocks for 84 management purposes. This is the first report of the nuclear genomes of the three study species 85 and provides useful baseline information for future genetic studies of these important species.

\section{Materials and methods}

88

Samples selected for the production of the microsatellite loci were derived from muscle tissue collected by the Northern Territory Department of Primary Industries and Fisheries and the Western Australian Department of Fisheries under Charles Darwin University Animal Ethics permit A13014. The L. johnii sample was a $210 \mathrm{~mm}$ male caught at $6 \mathrm{~m}$ depth in Darwin Harbour, Northern Territory, Australia (Middle Arm, 13058'0.24"E, 12³9'0.97"S) in 2013. The P. diacanthus sample was a $890 \mathrm{~mm}$ male caught in Fenton patches, Northern Territory, Australia $\left(130^{\circ} 42.084^{\prime} \mathrm{E}, 1^{\circ} 10.664^{\prime} \mathrm{S}\right)$ in 2013 . The L. laticaudis sample (WAM16-001) was a $419 \mathrm{~mm}$ male collected from East of the Lacepede Islands, Western Australia, Australia in 2013.

98 Genomic DNA from L. johnii and P. diacanthus was extracted using Qiagen DNeasy Blood \& 99 Tissue columns (Qiagen, Germantown, USA) following the manufacturer's instructions. Lethrinus laticaudis genomic DNA was extracted using a salting-out method as described in 
101 Broderick et al. (2011). Genomic DNA from all samples for testing the loci and further 102 genotyping was extracted using ISOLATE II Genomic DNA Kit (Bioline) following the 103 manufacturer's instructions. This resulted in $100 \mu \mathrm{L}$ of eluted DNA for each sample. All the 104 DNA extracts were quantified using the Qubit v3 (ThermoFisher) fluorometric method.

105

106

\subsection{Next-Generation Sequencing and primer selection}

107 The purified genomic DNA of L. johnii and P. diacanthus was prepared for direct shotgun 108 sequencing using the Iron ExpressTM fragment library kit and sequenced on an IonTorrent 109 Personal Genome Machine using an Ion318 chip (Life Technologies Corporation, Grand Island, $110 \mathrm{NY}$ ). The purified genomic DNA of L. laticaudis was sequenced on an Illumina ${ }^{\circledR}$ MiSeq as part 111 of a 2x300bp run at the Australian Genome Research Facility. Because two different NGS 112 platforms were used to scan the genomes of the three species we were able to compare their 113 performance for microsatellite design and to assess whether equivalent results were obtainable

114 from each platform. Lutjanus and Lethrinus genera and Sciaenidae are known to have genome 115 size comparable to other fish species (average size for Lutjanus $=1066 \mathrm{Mb}$, 116 Lethrinus $=1192 \mathrm{Mb}$, Sciaenidae $=753 \mathrm{Mb}$, Perciformes $=919 \mathrm{Mb}$; Gregory 2001). The 117 paired-end reads obtained with the MiSeq run were merged using FLASH source code (Magoč \& 118 Salzberg 2011) and their quality was checked in FastQC (Andrews 2010); the first 10 bp were 119 trimmed in Geneious v 9.0 (Drummond et al. 2011).

120 From the NGS data we looked for sequences longer than $300 \mathrm{bp}$ that contained a 121 microsatellite repeat that would be suitable for primer design. These sequences were checked for 122 microsatellite motifs and forward and reverse primers were designed using the software QDD2 123 beta (Meglécz et al. 2010). Sequences with target microsatellites and primers were then filtered 124 according to the following criteria: only pure repeats were selected; all dinucleotide repeats were 125 excluded; repeats greater than 8 were selected; loci with a predictive target sequence length 126 above $300 \mathrm{bp}$ were selected; primers with a distance less than $20 \mathrm{bp}$ from the repeat sequence 127 were excluded; and the PCR primers with a PCR_PRIMER_ALIGNSCORE equal or above 6 128 were excluded to discard primers with high alignment scores to the amplicon. A unique pair of 129 primers was selected for each locus. The PCR predicted sequences for all the loci were imported 130 into Geneious v 9.0 and blasted (MEGABLAST) against the NCBI GenBank database to check 131 if the microsatellites fell into coding regions. Sequences that would be homologous to any other 
132 NCBI sequence likely to be functional were excluded. All the primers were blasted against their

133 original genomic database built using the NGS reads. Only microsatellites with primers that had

134 one hit across the whole genome were kept for further steps to increase the chance of each

135 primer to amplify a unique sequence. As a final check point before wet laboratory work and to

136 make sure each pair of primers bound to the 5' and 3 ' ends of a unique sequence containing a

137 microsatellite we selected, containing microsatellites sequences and pairs of primers were

138 mapped de novo. For each species, we selected the 48 microsatellites that contained the best

139 quality repeats with the highest number of tri- tetra- or penta-nucleotide repeats possible and

140 with no small dinucleotide repeats between the primer and the microsatellite sequence to avoid

141 any noise that may interfere with scoring genotypes.

142 Forward primers were tagged on the $5^{\prime}$ end with the universal CAG sequence (5'-

143 CAGTCGGGCGTCATCA-3'). Inclusion of the 5'-tail will allow use of a CAG-tagged universal

144 primer in the PCR that is fluorescently labeled for detection on the sequencing machine

145 (Schuelke 2000). Additionally, a pig-tail (5'-GTTTCTT-3') was added to the 5' end of the

146 reverse primers to increase the accuracy of genotyping and ensure the consistency of the

147 amplicon size (Brownstein, Carpten \& Smith 1996). The resulting 48 pairs of primers were

148 synthetized by Integrated DNA Technologies (www.idtdna.com).

149

150

\subsection{Loci and primers testing}

151 For each species, the 48 pairs of primers were tested over a set of genomic DNA extracted from

152 eight individuals of the target species. The fluorescent dye labelling of PCR fragments in one

153 reaction was performed with three primers. Amplification reactions were carried out in a $8.8 \mu \mathrm{L}$

154 volume comprising $1 \mu \mathrm{L}$ of DNA, $4.84 \mu \mathrm{L}$ of $2 x$ Bioline Taq Mastermix, 4.4 pmol of locus-

155 specific forward CAG-tagged primer, 22 pmol of locus-specific reverse pig-tail-tagged primer

156 and 22 pmol of universal CAG primer labeled with a 6-FAM fluorescent dye. The heating cycle

157 parameters used for amplification were $95^{\circ} \mathrm{C}$ for $3 \mathrm{~min}, 37$ cycles of denaturation at $94^{\circ} \mathrm{C}$ for $15 \mathrm{~s}$,

158 annealing for $15 \mathrm{~s}$ at $57^{\circ} \mathrm{C}$ and elongation at $72^{\circ} \mathrm{C}$ for $60 \mathrm{~s}$. A final extension at $72^{\circ} \mathrm{C}$ for $30 \mathrm{~s}$ was

159 performed. Post-amplification, the PCR products were diluted with water 1:20. We added $2 \mu \mathrm{L}$

160 of these diluted PCR products to $10 \mu \mathrm{L}$ of Hi-Di formamide (ABI) and $0.05 \mu \mathrm{L}$ of GenScan-500

161 LIZ (ABI) size standard. Samples were denatured at $95^{\circ} \mathrm{C}$ for 3 min and sized on the ABI $3730 \times 1$

162 capillary sequencer (Applied Biosystems, Carlsbad, California, USA) using the conditions set 
163 down by the manufacturer. Chromatograms were analysed using Geneious v 9.0 (Drummond et 164 al. 2011).

165 Criteria used to select the best loci among the 48 tested for each species included the 166 amplification success rate, peak intensity, the presence or absent of stutter peaks, the 167 polymorphism of the loci, the number of alleles and heterozygosity. The best loci were 168 individually tested against a further 23 samples of the target species.

169

170

171 In order to reduce the cost and time of genotyping for further genetic studies, the newly designed 172 microsatellites were combined into multiplex panels of 2 to 4 loci. The panels were set up based 173 on the microsatellite allele-size range. The primers for all the loci of each panel were combined 174 in a single PCR to allow the amplification of several microsatellite loci at the same time. When 175 allele-size ranges overlapped, alternate dyes were employed to allow the discrimination of each 176 locus on the chromatograms. Each of the four ABI dyes FAM, VIC, NED and PET were tailed 177 with a unique M13 primer: M13F (5'-TTTCCCAGTCACGACGTTG-3'), M13V (5' 178 GCGGATAACAATTTCACACAGG-3'), M13N (5'-TAAAACGACGGCCAGTGC-3') and 179 M13P (5'-CACAGGAAACAGCTATGACC-3'). The 5' end of the forward primer for the locus 180 was synthetized with the corresponding M13 tail to allow fluorescent labeling of PCR product 181 using a 3-primer PCR protocol as described above (Schuelke 2000). Several multiplex trials were 182 conducted to find the best combination of loci with the optimal concentration of primers and 183 PCR parameters. Primer pairs that failed to amplify within a multiplex were removed from the 184 panels and further optimization focused on the remaining primer pairs. For each species, the multiplex trials were all evaluated against 8 samples that were the same for those used in the 23 samples above, allowing the consistency to be checked across the templates.

187

\subsection{Genetic variability and population genetics}

In order to test if the herein developed microsatellites would be useful to discriminate fish stocks across northern Australia we collected samples from two locations in the Northern Territory and one location in Western Australia and assayed their population structure. For each of the three species, samples were collected from Camden Sound (Western Australia, Australia), Wadeye 
194 bottom of the Gulf of Carpentaria (Northern Territory, Australia) (Figure 1). Fourteen L. johnii, 195 eighteen $P$. diacanthus and twenty-eight L. laticaudis were collected from Camden Sound 196 (Western Australia, Australia); twenty-nine L.johnii, twenty-five P. diacanthus and twenty197 seven L. laticaudis were collected from Wadeye (Northern Territory, Australia); twenty-five 198 L. johnii, twenty-nine $P$. diacanthus and twenty-nine L. laticaudis were collected from Vanderlin 199 Islands (Northern Territory, Australia). All individuals were assayed as part of the multiplex 200 panels or PCR pooled. The multiplex PCR were comprised of 2-5 $\mu \mathrm{L}$ of DNA depending on the 201 samples (approximately $20 \mathrm{ng}$ total), $8 \mu \mathrm{L}$ of $2 \mathrm{x}$ Bioline Taq Mastermix and $4 \mu \mathrm{L}$ of panel 202 primer mix containing various quantities of primers as described in Table 1. Concentrations of 203 the different primers were adjusted to obtain homogenous PCR products revealed by similar 204 intensity chromatogram peaks for each of the dyes within each panel. The heating cycle 205 parameters, sizing of the alleles and chromatogram analyses were conducted using the same 206 method as stated above.

207 The obtained datasets were statistically evaluated. The potential for null alleles, large 208 allele dropout and stuttering to interfere with scoring accuracy was evaluated for each 209 microsatellite locus in each sample using Microchecker v.2.2.3 (Van Oosterhout et al. 2004). The 210 software Arlequin 3.5.2.2 (Excoffier \& Lischer 2010) was used to calculate the number of alleles $211(A)$, expected $\left(H_{e}\right)$ and observed $\left(H_{o}\right)$ heterozygosity and conduct exact tests of conformance of 212 genotypic proportions to Hardy-Weinberg equilibrium expectations. Estimation of probability 213 values $\left(P_{H W}\right)$ employed a Monte Carlo Markov Chain (MCMC) of $10^{5}$ steps and 5.104 214 dememorization. Genotypic equilibrium between pairs of microsatellites (linkage disequilibrium) 215 was tested in Arlequin with 10,000 permutations. Fixation indices $\left(\mathrm{F}_{\mathrm{ST}}\right)$ between pairs of sample 216 localities were estimated as implemented in Arlequin to identify possible spatial boundaries 217 among sample locations.

218

\section{3. Results and Discussion}

220

221 The IonTorrent sequencing technology allowed the production of longer reads (range 8-620 bp) 222 compared to the Illumina MiSeq that produced $300 \mathrm{bp}$ reads fixed by the method. The paired-end 223 reads of the MiSeq run were merged to increase their length to 300-575 bp and allow the 224 detection of at least $300 \mathrm{bp}$ length sequences that may contain a microsatellite locus. This 
225 resulted in 1,169,198 reads, which is less than what was used to select microsatellite loci in the

226 first two species. The MiSeq run produced higher quality sequences than the IonTorrent (Phred

227 score: 36 vs 29). Quality profiles along the IonTorrent reads showed that the quality of the

228 sequencing decreased with length meaning that the end of the longer reads ( $>325 \mathrm{bp}$ ) had a

229 lower quality then at their start. QDD Pipe1 detected between 110,000 and 170,000 sequences

230 containing a microsatellite sequence depending on the species (Table 2). This number was

231 independent of the type of NGS platform used. From those sequences QDD Pipe2 removed the

232 low complexity sequences (no BLAST to itself), putative minisatellites (short sequences of

233 repeated nucleotides) and sequences that had BLAST hit to other sequences to only keep the

234 singletons and unique consensus sequences. QDD Pipe3 designed primers for all QDD Pipe2

235 output reads. The resulting number of sequences that contained a microsatellite sequence and the

236 corresponding primers were given in the final output of QDD pipeline, and varied between

23720,000 and 30,000 depending on the species (Table 2). After applying the filtering criteria

238 described previously, 97 potentially amplifiable microsatellite reads were found for

239 P. diacanthus, 121 for L. johnii and 103 for L. laticaudis. From those microsatellite reads, we

240 selected the ones with the smallest number of repeats but greater than 8 and eliminated those

241 with small repeats between the primer and the microsatellite to reach 48 microsatellite loci per

242 species being ultimately tested in the laboratory.

243 The testing of 144 primer pairs resulted in the selection of 34 polymorphic loci that could

244 be reliably scored and showed consistent amplification success. We selected a final set of 10 loci

245 for L. johnii, 11 loci for P. diacanthus and 13 loci for L. laticaudis (Table 3). Multiplex panels of

246 microsatellites were developed for the two species L.johnii and P. diacanthus, and the

247 optimization of each panel resulted in the efficient assay and unambiguous scoring of

248 microsatellites in the two species. Although the M13 labeling system worked very well for

249 L. johnii and P. diacanthus it did not amplify successfully as part of PCR multiplexes for

250 L. laticaudis. The reasons why it did not work well for this species are still unclear as the quality

251 of the DNA was even across the three species and the same protocol was followed. However, in-

252 house experiments showed that lengthening the labeled forward primer might facilitate the PCR

253 reaction when multiplexing several loci. Direct labeling of the forward primer may also be

254 another option for multiplexing a large number of loci. For L. laticaudis, the loci were all 
255 amplified in individual PCR with the CAG labeling system as described above. The resulting 256 PCR products were then pooled according to the panels described in Table 1 before the ABI run. 257 Genotypes from 10 microsatellites were obtained from multiplexed PCR for 68 258 individuals of L. johnii. There was $1.91 \%$ missing data and the number of alleles for each locus 259 varied between 4 and 23 (Table 3). Microchecker indicated the possible occurrence of null 260 alleles at location Wa for locus LujO12 and at CS for locus LujO18 with possible stuttering or 261 scoring errors for the latter. There were only 3/45 significant tests for linkage disequilibrium 262 between pairs of loci (Luj094 x Luj051, Luj051 x Luj091 and Luj076 x Luj082) and there was no 263 deviation from Hardy-Weinberg equilibrium (HWE) detected. Heterozygosity was moderate to 264 high for all loci (mean overall loci $0.707+/-0.200)$ and generally similar to expectations (around 2650.7 for marine fish, DeWoody \& Avise 2000). Genotypes from 11 microsatellites were obtained 266 from multiplexed PCR for 72 individuals of $P$. diacanthus. There was $2.77 \%$ missing data and 267 the number of alleles for each locus varied between 5 and 19 (Table 3). Microchecker indicated 268 the possible occurrence of null alleles at location CS for locus Prd068 and at VI for locus $269 \operatorname{Prd012}$. There were only 3/55 significant tests for linkage disequilibrium between pairs of loci 270 (Prd046 x Prd018, Prd020 x Prd018 and Prd018 x Prd045) and overall deviations from Hardy271 Weinberg equilibrium (HWE) were detected at two loci Prd023 (p-value=0.034) and Prd018 (p272 value $=0.008$ ). Heterozygosity was variable and with an overall mean lower than for L. johnii $273(0.673+/-0.185)$. Genotypes from 10 microsatellites were obtained from pooled post PCR 274 products for 84 individuals of L. laticaudis as 3 of our developed microsatellites did not 275 amplified consistently in all the samples. There was $2.61 \%$ missing data and the number of 276 alleles for each locus varied between 9 and 22 (Table 3). Microchecker indicated the possible 277 occurrence of null alleles at location CS for loci Lel033 and Lel012. There were 6/78 significant 278 tests for linkage disequilibrium between pairs of loci (Lel040 x Lel012, Lel040 x Lel032, Lel011 279 x Lel027, Lel012 x Lel032, Lel012 x Lel013 and Lel028 x Lel027) and overall deviations from 280 Hardy-Weinberg equilibrium (HWE) were detected at a single locus Lel012 (p-value=0.005). 281 Heterozygosity was high and with an overall mean higher than for the two other species $(0.834$ $282+/-0.078)$.

283 A pattern of genetic differentiation with low but significant population-pairwise $F_{\mathrm{ST}}$ 284 (range 0.007-0.017) was observed in the three species (Table 4). These levels of differentiation 285 are similar in magnitude to those reported for other marine fish species with potentially high 
286 gene flow (O'Reilly et al. 2004). The three species also presented different patterns of structure

287 between the three locations. L. laticaudis presented pairwise differentiation between Western 288 Australia location (CS) and the two other Northern Territory locations (VI and Wa). Similarly, 289 P. diacanthus presented a structure between the two most distant populations of CS and VI 290 whereas CS and Wa remained undifferentiated. By contrast, L. johnii did not present structure 291 between Western Australia and Northern Territory. However, VI and Wa in the Northern 292 Territory had a significant pairwise $\mathrm{F}_{\text {ST. }}$. Pairwise $\mathrm{F}_{\mathrm{ST}}$ values were low in all comparisons for the 293 three species (range 0.001-0.017) as commonly reported in marine species and, 4 out of 9 294 pairwise comparisons were significant meaning the microsatellites we developed can accurately 295 detect population differentiation across Northern Australia and may be used for genetic structure 296 studies and stock identification.

297

\section{4. Conclusion}

299

300 In conclusion, we applied the direct sequencing of a genomic library approach to develop 301 microsatellite loci and it resulted in a significant reduction in laboratory effort compared to 302 traditional protocols for microsatellite discovery (cloning and Sanger sequencing). Merged paired-end reads from the MiSeq platform demonstrated higher quality of reads than the IonTorrent single reads. From the 1-1.5 million raw reads, we selected a reduced number of loci to test (48) and successfully amplified satisfactory polymorphic loci for 10 to 13 of them depending on the species. However, the NGS data revealed the potential for hundreds to thousands of potentially amplifiable microsatellites to be discovered. The microsatellites characterized in this work will be available to explore the population genetics and stock structure of these highly valuable species.

\section{Acknowledgements}

312 The authors thank staff from the Western Australian Department of Fisheries and the Northern 313 Territory Department of Primary Industries as well as Indigenous rangers for sample collection. 314 We extend our thanks to Samuel Williams at the Molecular Fisheries laboratory and Sean Corley 315 at the Animal Genetics Laboratory for their assistance with laboratory work. 


\section{References}

318

319

320

321

322

323

324

325

326

327

328

329

330

331

332

333

334

335

336

337

338

339

340

341

342

343

344

345

346

347

348

349

350

351

352

353

354

355

356

357

358

359

360

361

362

Abdelkrim J, Robertson BC, Stanton J-AL, and Gemmell NJ. 2009. Fast, cost-effective development of species-specific microsatellite markers by genomic sequencing. Biotechniques 46:185.

Allen GR, Swainston R, and Ruse J. 1997. Marine fishes of tropical Australia and south-east Asia. Perth: Western Australian Museum.

Andrews S. 2010. FastQC: a quality control tool for high throughput sequence data. Available online at: http://www.bioinformatics.babraham.ac.uk/projects/fastqc

Ayvazian S, Chatfield B, and Keay I. 2004. The age, growth, reproductive biology and stock assessment of grass emperor, Lethrinus laticaudis in Shark Bay, Western Australia. Department of Fisheries, Research Division, Westrern Australian Marine Research Laboratories.

Bowtell B. 1995. Heed jewfish warnings. Fish and Boat July.

Bowtell B. 1998. Huge schools of black jew about. Fish and Boat July.

Broderick D, Ovenden J, Buckworth R, Newman S, Lester R, and Welch D. 2011. Genetic population structure of grey mackerel Scomberomorus semifasciatus in northern Australia. Journal of Fish Biology 79:633-661.

Brownstein MJ, Carpten JD, and Smith JR. 1996. Modulation of non-templated nucleotide addition by Taq DNA polymerase: primer modifications that facilitate genotyping. Biotechniques 20:1004-1006.

Castoe TA, Poole AW, Gu W, Jason de Koning A, Daza JM, Smith EN, and Pollock DD. 2010. Rapid identification of thousands of copperhead snake (Agkistrodon contortrix) microsatellite loci from modest amounts of 454 shotgun genome sequence. Molecular Ecology Resources 10:341-347.

Coleman APM. 2004. The national recreational fishing survey: the Northern Territory. Department of Business Industry and Resource Development. Fishery Report 72.

DeWoody J, and Avise J. 2000. Microsatellite variation in marine, freshwater and anadromous fishes compared with other animals. Journal of Fish Biology 56:461-473.

Drummond A, Ashton B, Buxton S, Cheung M, Cooper A, Duran C, Field M, Heled J, Kearse M, and Markowitz S. 2011. Geneious v5. 4.

Excoffier L, and Lischer HE. 2010. Arlequin suite ver 3.5: a new series of programs to perform population genetics analyses under Linux and Windows. Molecular ecology resources 10:564567.

Fernandez-Silva I, Whitney J, Wainwright B, Andrews KR, Ylitalo-Ward H, Bowen BW, Toonen RJ, Goetze E, and Karl SA. 2013. Microsatellites for next-generation ecologists: a postsequencing bioinformatics pipeline. PLoS one 8:e55990.

Gardner MG, Fitch AJ, Bertozzi T, and Lowe AJ. 2011. Rise of the machines-recommendations for ecologists when using next generation sequencing for microsatellite development. Molecular Ecology Resources 11:1093-1101.

Gregory TR. 2001. Animal genome size database. Available at http://www.genomesize.com. Grubert M, Saunders T, Martin JM, Lee H, and Walters CJ. 2013. Stock Assessments of Selected Northern Territory Fishes. Northen Territory Government. Fishery Report 110.

Grubert MA, Kuhl PJ, and Penn JW. 2010. Ecological risk assessment. Northern Territory coastal line fishery. Norther Territory Department of Resources. Fishery Report 103.

Lenanton R, and Potter I. 1987. Contribution of estuaries to commercial fisheries in temperate Western Australia and the concept of estuarine dependence. Estuaries 10:28-35. 
363 Magoč T, and Salzberg SL. 2011. FLASH: fast length adjustment of short reads to improve 364 genome assemblies. Bioinformatics 27:2957-2963.

365 Meglécz E, Costedoat C, Dubut V, Gilles A, Malausa T, Pech N, and Martin J-F. 2010. QDD: a 366 user-friendly program to select microsatellite markers and design primers from large sequencing 367 projects. Bioinformatics 26:403-404.

368 O'Reilly P, Canino M, Bailey K, and Bentzen P. 2004. Inverse relationship between $\mathrm{F}_{\mathrm{ST}}$ and 369 microsatellite polymorphism in the marine fish, walleye pollock (Theragra chalcogramma): implications for resolving weak population structure. Molecular Ecology 13:1799-1814. Peters MB, Ovenden JR, Broderick D, Lance SL, Hagen C, and Glenn TC. 2009. Fifteen microsatellite loci for the jungle perch, Kuhlia rupestris. Molecular Ecology Resources 9:14671469.

374 Phelan MJ. 2001. Sciaenid aggregations in northern Australia: an example of successful 375 outcomes through collaborative research. Fisheries Centre Research Reports: 100.

Rao TA, Mohan RL, Chakraborty S, Murty VS, Nair KS, Vivekanandan E, and Raje S. 1992. Stock assessment of sciaenid resources of India. Indian Journal of Fisheries 39:85-103.

Rutherford ES, Tilmant JT, Thue EB, and Schmidt TW. 1989. Fishery harvest and population dynamics of spotted seatrout, Cynoscion nebulosus, in Florida Bay and adjacent waters. Bulletin of Marine Science 44:108-125.

Saunders T, Newman S, Keag M, and Errity C. 2014a. Golden Snapper Lutjanus johnii. Status of key Australian fish stocks reports 2014. Fisheries Research and Development Corporation. Canberra. 523-529.

384 Saunders T, Roelofs A, Newman S, and Errity C. 2014b. Black jewfish Protonibea diacanthus. 385 Status of key Australian fish stocks reports 2014. Fisheries Research and Development 386 Corporation. Canberra. 344-350.

387 Schuelke M. 2000. An economic method for the fluorescent labeling of PCR fragments. Nature 388 Biotechnology 18:233-234.

389 True CD, Loera AS, and Castro NC. 1997. Technical Notes: Acquisition of Broodstock of 390 Totoaba macdonaldi: Field Handling, Decompression, and Prophylaxis of an Endangered 391 Species. The Progressive Fish-Culturist 59:246-248.

392 Van Oosterhout C, Hutchinson WF, Wills DP, and Shipley P. 2004. MICRO-CHECKER: 393 software for identifying and correcting genotyping errors in microsatellite data. Molecular 394 Ecology Notes 4:535-538. 


\section{Table $\mathbf{1}$ (on next page)}

Technical details on the multiplex polymerase chain reaction (PCR) and post-PCR pooled products of microsatellite loci in Lutjanus johnii, Protonibea diacanthus and Lethrinus laticaudis.

Included in the table are the multiplex PCR panels for $L$. johnii, $P$. diacanthus and post-PCR pooled products panels for $L$. laticaudis, primer mix quantities per reaction $(\mu L)$ within each multiplex and fluorescent dye labels used for each locus in the PCR reactions. 


\begin{tabular}{|c|c|c|c|c|}
\hline Species & Panel & Microsatellite & Quantity $(\mu \mathrm{L})$ & ABI Dye \\
\hline \multirow[t]{10}{*}{ Lutjanus johnii } & \multirow[t]{4}{*}{1} & Luj094 & 0.2 & FAM \\
\hline & & Luj068 & 0.6 & PET \\
\hline & & Luj027 & 0.6 & FAM \\
\hline & & Luj076 & 0.6 & VIC \\
\hline & \multirow[t]{4}{*}{2} & Luj051 & 0.2 & VIC \\
\hline & & Luj090 & 0.4 & FAM \\
\hline & & Luj114 & 0.6 & VIC \\
\hline & & Luj091 & 0.6 & PET \\
\hline & \multirow[t]{2}{*}{3} & Luj072 & 0.7 & FAM \\
\hline & & Luj082 & 0.7 & VIC \\
\hline \multirow[t]{11}{*}{ Protonibea diacanthus } & \multirow[t]{4}{*}{1} & $\operatorname{Prd012}$ & 0.2 & PET \\
\hline & & $\operatorname{Prd023}$ & 0.2 & VIC \\
\hline & & $\operatorname{Prd044}$ & 0.2 & FAM \\
\hline & & $\operatorname{Prd042}$ & 0.2 & NED \\
\hline & \multirow[t]{4}{*}{2} & $\operatorname{Prd018}$ & 0.4 & NED \\
\hline & & $\operatorname{Prd045}$ & 0.2 & PET \\
\hline & & $\operatorname{Prd046}$ & 0.2 & FAM \\
\hline & & $\operatorname{Prd020}$ & 0.2 & PET \\
\hline & \multirow[t]{3}{*}{3} & $\operatorname{Prd036}$ & 0.2 & VIC \\
\hline & & $\operatorname{Prd049}$ & 0.2 & FAM \\
\hline & & $\operatorname{Prd024}$ & 0.2 & PET \\
\hline \multirow[t]{13}{*}{ Lethrinus laticaudis } & \multirow[t]{4}{*}{1} & Lel033 & - & NED \\
\hline & & Lel012 & - & PET \\
\hline & & Lel040 & - & VIC \\
\hline & & Lel011 & - & FAM \\
\hline & \multirow[t]{4}{*}{2} & Lel032 & - & NED \\
\hline & & Lel028 & - & PET \\
\hline & & Lel041 & - & VIC \\
\hline & & Lel013 & - & FAM \\
\hline & \multirow[t]{4}{*}{3} & Lel036 & - & NED \\
\hline & & Lel039 & - & PET \\
\hline & & Lel044 & - & VIC \\
\hline & & Lel047 & - & FAM \\
\hline & 4 & Lel027 & - & FAM \\
\hline
\end{tabular}

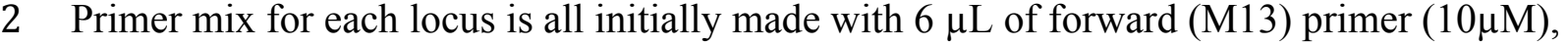
$330 \mu \mathrm{L}$ of reverse primer $(10 \mu \mathrm{M}), 30 \mu \mathrm{L}$ of $\mathrm{M} 13$ labeled dye $(10 \mu \mathrm{M})$ and $84 \mu \mathrm{L}$ of water. The 
4 primer mixes of each panel are mixed in the proportion given in the table with water added to 5 reach $4 \mu \mathrm{L}$. 


\section{Table 2 (on next page)}

Next-generation sequencing and bioinformatics details obtained from FastQC software (Andrews, 2010) and QDD pipeline (Meglécz et al., 2010) for Lutjanus johnii, Protonibea diacanthus and Lethrinus laticaudis. 


\begin{tabular}{|c|c|c|c|}
\hline & Lutjanus johnii & Protonibea diacanthus & Lethrinus laticaudis \\
\hline Genomic DNA extraction & Qiagen DNeasy & Qiagen DNeasy & Salting out \\
\hline NGS Technology & IonTorrent | Ion318Chip & IonTorrent | Ion318Chip & Illumina ${ }^{\circledR}$ MiSeq \\
\hline Library preparation & Iron ExpressTM & Iron ExpressTM & \\
\hline Type of reads & Single reads & Single reads & Paired-end reads \\
\hline Number of reads & $1,374,891$ & $1,587,789$ & $2,800,640$ \\
\hline Merged reads & - & - & $1,169,198$ \\
\hline Reads length & $8-620$ & $8-618$ & 300 \\
\hline Merged reads length & - & - & $300-575$ \\
\hline \multicolumn{4}{|l|}{ FASTQC } \\
\hline$\% \mathrm{GC}$ & 41 & 42 & 39 \\
\hline Sequence quality $<$ Phred 20 & yes at positions $>325 \mathrm{bp}$ & yes at positions $>350 \mathrm{bp}$ & no \\
\hline Per sequence quality - Phred score & 29 & 29 & 36 \\
\hline Sequence length distribution & peak at $350 \mathrm{bp}$ & peak at $350 \mathrm{bp}$ & plateau at $520-540 \mathrm{bp}$ \\
\hline \multicolumn{4}{|l|}{ QDD2 } \\
\hline \multicolumn{4}{|c|}{ QDD2 pipe 1 - Sequence preparation and microsatellite detection } \\
\hline Number sequence length $\geq 80 \mathrm{bp}$ & $1,235,685$ & $1,405,082$ & $1,169,198$ \\
\hline Number sequence with microsatellite & $109,641(8.9 \%)$ & $167,702(11.9 \%)$ & $130,269(11.1 \%)$ \\
\hline \multicolumn{4}{|l|}{ QDD2 pipe 2 - Sequence similarity detection } \\
\hline Total \# input sequences & 109,641 & 167,702 & 130,269 \\
\hline Numer of unique consensus sequences & 18,978 & N/A & N/A \\
\hline Number of singleton sequences & 49,122 & N/A & N/A \\
\hline Number of reads in output & 68,100 & 63,789 & 69,714 \\
\hline \multicolumn{4}{|l|}{ QDD2 pipe 3 - Primer design } \\
\hline Total number of input sequences & 68,100 & 63,789 & 69,714 \\
\hline Total number of sequences with target MS & 67,461 & 63,785 & 69,714 \\
\hline Total number of sequences with primers & 29,485 & 20,233 & 19,867 \\
\hline \multicolumn{4}{|l|}{ Filtering QDD output } \\
\hline Total \# input sequences & 29,485 & 20,233 & 19,867 \\
\hline Total \# sequences after filtering criteria & 121 & 97 & 103 \\
\hline
\end{tabular}


Figure 1

Location of the three sampling sites across northern Australia.

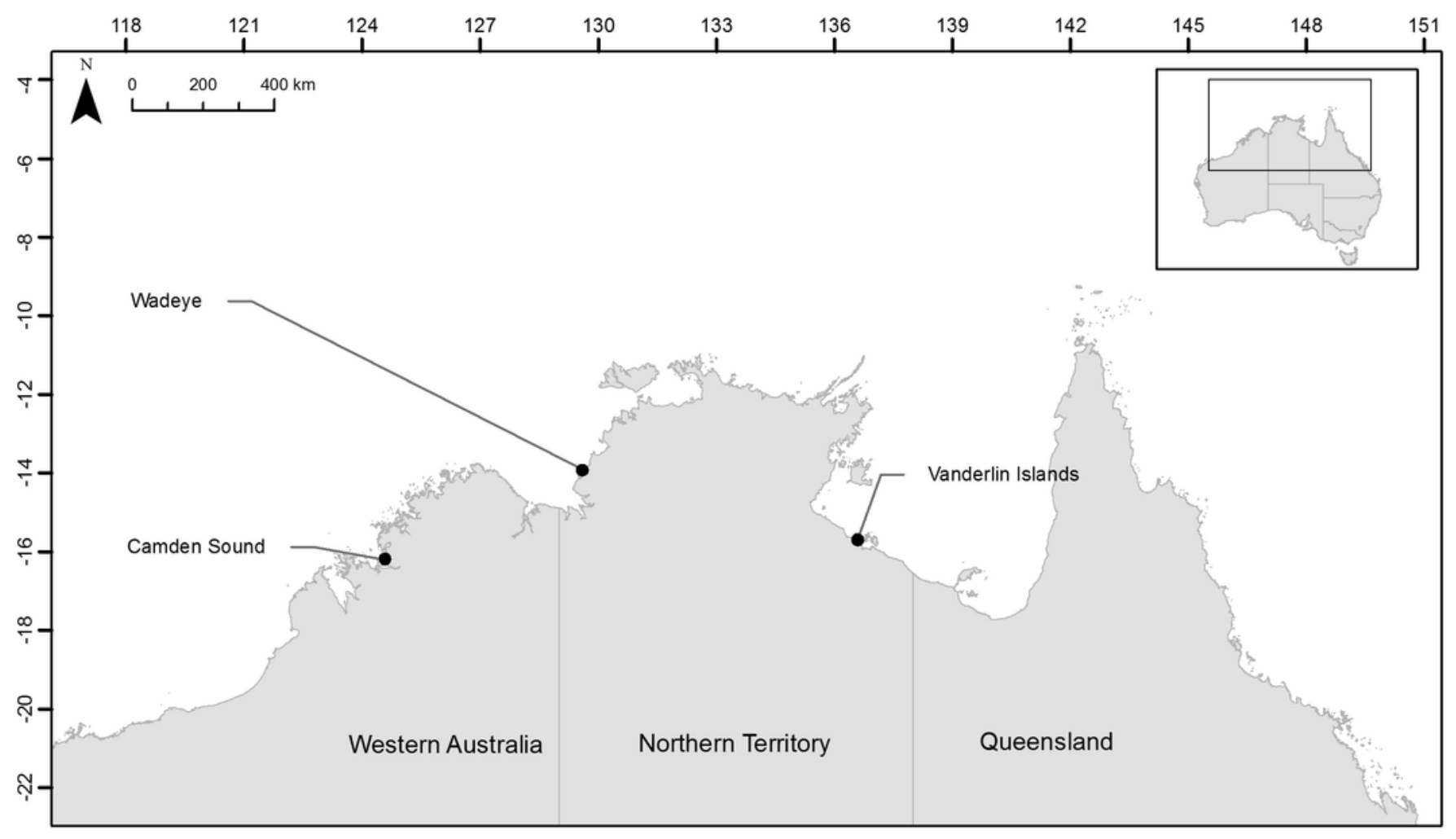




\section{Table 3(on next page)}

Characteristics of the 34 microsatellite markers developed in Lutjanus johnii, Protonibea diacanthus and Lethrinus laticaudis.

$n$ is the sample size, \#A is the number of alleles at each loci, $H_{E}$ is the expected heterozygosity, $H_{O}$ is the observed heterozygosity and $P_{H W}$ is the $p$-value of the exact tests of conformance of genotypic proportions to Hardy-Weinberg equilibrium expectations. 


\begin{tabular}{|c|c|c|c|c|c|c|c|c|c|c|c|}
\hline Species & Locus & & er sequences (5'-3') (Fluorescent label) & Repeat motif & $\begin{array}{l}\text { GenBank } \\
\text { accession } \\
\text { no. }\end{array}$ & $\mathrm{n}$ & $\begin{array}{l}\text { Allele } \\
\text { size } \\
\text { range } \\
(\mathrm{bp})\end{array}$ & $\# \mathrm{~A}$ & $\mathrm{H}_{\mathrm{O}}$ & $\mathrm{H}_{\mathrm{E}}$ & $\mathrm{P}_{\mathrm{HW}}$ \\
\hline \multirow[t]{20}{*}{$\begin{array}{l}\text { Lutjanus } \\
\text { johnii }\end{array}$} & Luj027 & $\mathrm{F}:$ & CTGGGCCACACTGATAAAGC (FAM) & $(\mathrm{AGC}) 9$ & KX387441 & 68 & $152-179$ & 8 & 0.309 & 0.306 & 0.606 \\
\hline & & $\mathrm{R}:$ & GGCTCTGAACCTGGGAGATT & & & & & & & & \\
\hline & Luj094 & F: & TCTCAGAGGGTTTGATGCAG (FAM) & $(\mathrm{AATC}) 9$ & KX387437 & 68 & $223-239$ & 4 & 0.426 & 0.470 & 0.205 \\
\hline & & $\mathrm{R}:$ & CTTTGGCGCTTTCTATCAGC & & & & & & & & \\
\hline & Luj076 & F: & CGGGTCGAGTCTGTTTGTGT (VIC) & $(\mathrm{AAG}) 15$ & KX387436 & 66 & $200-233$ & 10 & 0.818 & 0.811 & 0.289 \\
\hline & & $\mathrm{R}:$ & CTTCAGACGGATTAGCAGCA & & & & & & & & \\
\hline & Luj068 & F: & CCTAGGGTGTCAGTGTCAGTCA (PET) & $(\mathrm{AAAG}) 20$ & KX387435 & 68 & $174-258$ & 18 & 0.882 & 0.936 & 0.212 \\
\hline & & $\mathrm{R}:$ & TGCCTGTATGTTCTCTTGAGC & & & & & & & & \\
\hline & Luj090 & F: & ATCCTAATGCATCGTGCTTG (FAM) & $(\mathrm{AGC}) 17$ & KX387444 & 68 & $194-278$ & 23 & 0.868 & 0.922 & 0.146 \\
\hline & & $\mathrm{R}:$ & GGCATGTTCTATTGAGGTTGG & & & & & & & & \\
\hline & Luj051 & F: & TGCAGAGCAACAGAACAACAC (VIC) & $(\mathrm{ACTG}) 10$ & KX387440 & 67 & $172-192$ & 6 & 0.687 & 0.596 & 0.377 \\
\hline & & $\mathrm{R}:$ & CACCTTGCGTTTGCAGTCT & & & & & & & & \\
\hline & Luj114 & F: & CCATAACTGCTGTTCTGTATCTGG (VIC) & $(\mathrm{AGC}) 9$ & KX387442 & 68 & $276-314$ & 11 & 0.794 & 0.739 & 0.725 \\
\hline & & $\mathrm{R}:$ & AATACGGCAGATCTCGGGTT & & & & & & & & \\
\hline & Luj091 & F: & TCATTCCCAGGAGCTCAAAT (PET) & $(\mathrm{ACAG}) 12$ & KX387438 & 64 & $219-279$ & 12 & 0.813 & 0.782 & 0.499 \\
\hline & & $\mathrm{R}:$ & AATCGTCACTTTCGACCCAC & & & & & & & & \\
\hline & Luj072 & F: & ACTCGAAGAACACAGCCCAC (FAM) & $(\mathrm{AGC}) 9$ & KX387443 & 65 & $192-204$ & 5 & 0.738 & 0.675 & 0.052 \\
\hline & & $\mathrm{R}:$ & CACATTTGAATCCTTGCTGG & & & & & & & & \\
\hline & Luj082 & F: & AAGTACATCGGAGGGCTGAG (VIC) & $(\mathrm{ACGAT}) 12$ & KX387439 & 65 & $220-275$ & 12 & 0.800 & 0.833 & 0.548 \\
\hline & & $\mathrm{R}:$ & TGTTATCAAAGTTCACCGATACAAA & & & & & & & & \\
\hline \multirow[t]{15}{*}{$\begin{array}{l}\text { Protonibea } \\
\text { diacanthus }\end{array}$} & $\operatorname{Prd044}$ & F: & ACAAAGTTTCCTCCTCTGGC (FAM) & $(\mathrm{AAG}) 13$ & KX387452 & 71 & $181-211$ & 11 & 0.746 & 0.804 & 0.324 \\
\hline & & $\mathrm{R}:$ & CACGTTCCATCTTTATTTATTTGC & & & & & & & & \\
\hline & $\operatorname{Prd023}$ & F: & TCGTGTGAACACTTTGATGC (VIC) & (ATC)11 & KX387448 & 72 & 292-316 & 9 & 0.875 & 0.847 & 0.034 \\
\hline & & $\mathrm{R}:$ & CTCGTCTCTGCTCTTGGTCC & & & & & & & & \\
\hline & $\operatorname{Prd} 042$ & F: & TACCTTTGAGATGCGAGCG (NED) & $(\mathrm{AGC}) 12$ & KX387451 & 72 & $230-248$ & 7 & 0.694 & 0.698 & 0.526 \\
\hline & & $\mathrm{R}:$ & GTCAAAGCCATCAATCCAGC & & & & & & & & \\
\hline & $\operatorname{Prd012}$ & F: & AGGCTGTTTGAACTGCAGGG (PET) & $(\mathrm{AAAG}) 20$ & KX387445 & 64 & $195-271$ & 19 & 0.828 & 0.898 & 0.324 \\
\hline & & $\mathrm{R}:$ & CATGCTGAGCAATATGTGGG & & & & & & & & \\
\hline & $\operatorname{Prd046}$ & F: & TCATCCTGAGTTTGTGCTGG (FAM) & $(\mathrm{AGC}) 9$ & KX387454 & 72 & $224-236$ & 5 & 0.347 & 0.351 & 0.693 \\
\hline & & $\mathrm{R}:$ & CATGAGTAAGCAGAGCGTGG & & & & & & & & \\
\hline & $\operatorname{Prd018}$ & F: & ATGAACGGCATCAGTCAGC (NED) & (ACAG)9 & KX387446 & 68 & 179-207 & 8 & 0.662 & 0.784 & 0.008 \\
\hline & & $\mathrm{R}:$ & CGTCTGATAAACAGCACTGCC & & & & & & & & \\
\hline & $\operatorname{Prd020}$ & F: & CAATGTTCTGCAAGAGCTGC (PET) & $(\mathrm{ATC}) 11$ & KX387447 & 71 & $189-216$ & 10 & 0.746 & 0.713 & 0.837 \\
\hline & & $\mathrm{R}:$ & TCAAATGTCAAAGTCCAGTCC & & & & & & & & \\
\hline & $\operatorname{Prd} 045$ & F: & GTCTATCCATGTTCCAGCCC (PET) & $(\mathrm{ATCC}) 11$ & KX387453 & 64 & $279-309$ & 8 & 0.672 & 0.639 & 0.400 \\
\hline
\end{tabular}




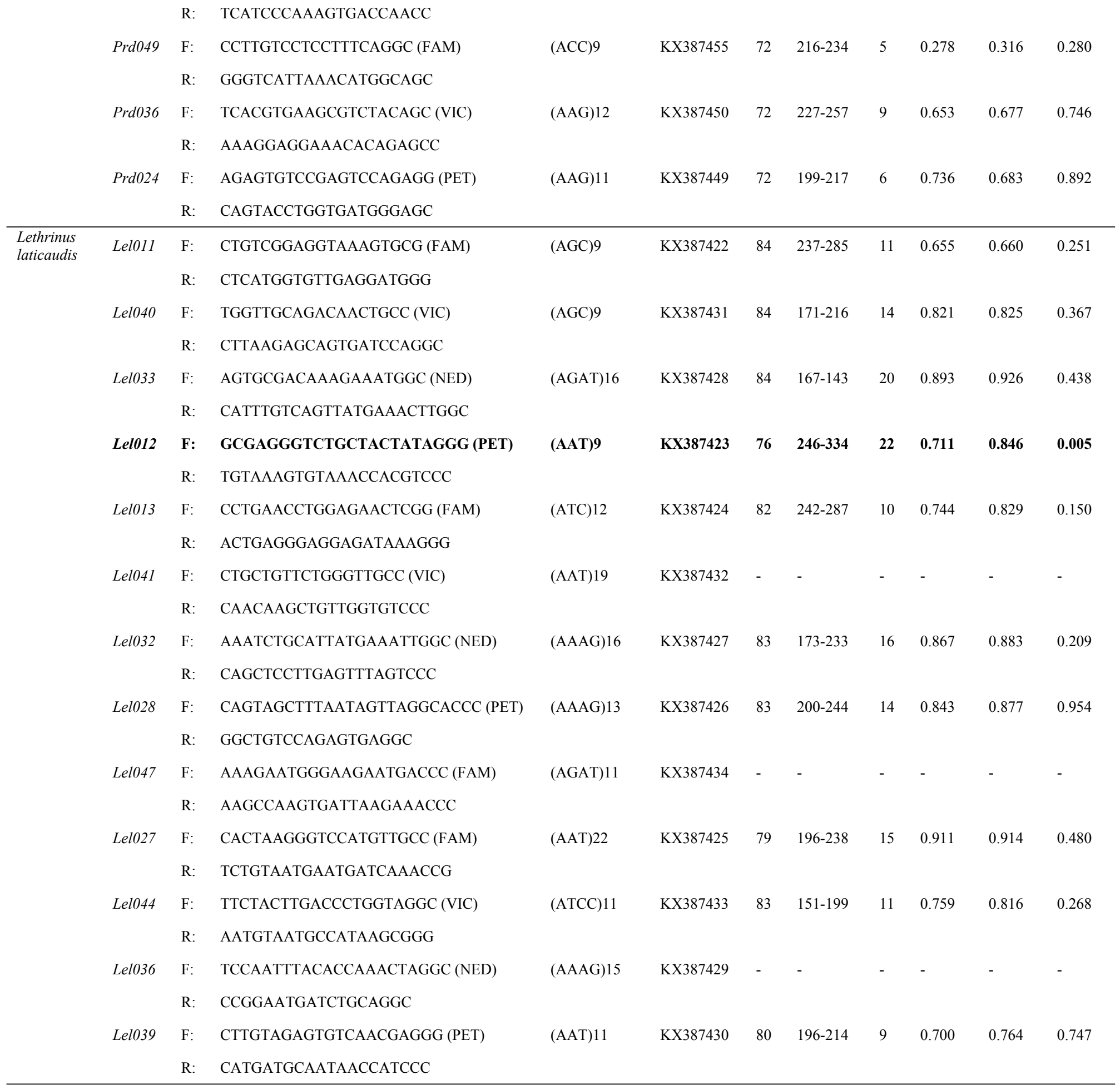




\section{Table 4(on next page)}

Pairwise $\mathrm{F}_{\mathrm{ST}}$ estimates for Lutjanus johnii, Protonibea diacanthus, and Lethrinus laticaudis for three locations.

Estimates are based on 10 microsatellite data from 68 individuals of $L$. johnii, 11 microsatellite data from 72 individuals of $P$. diacanthus and 10 microsatellite data from 84 individuals of $L$. laticaudis among the three sampling locations Camden Sound (CS), Wadeye (Wa) and Vanderlin Islands (VI). The comparisons that differed significantly from zero $(p<0.05)$ are shaded in grey. 


\begin{tabular}{lllllll} 
& \multicolumn{2}{c}{ Lutjanus johnii } & \multicolumn{2}{c}{ Protonibea diacanthus } & \multicolumn{2}{c}{ Lethrinus laticaudis } \\
\cline { 2 - 7 } & $\mathrm{F}_{\mathrm{ST}}$ & $\mathrm{p}$-value & $\mathrm{F}_{\mathrm{ST}}$ & $\mathrm{p}$-value & $\mathrm{F}_{\mathrm{ST}}$ & $\mathrm{p}$-value \\
\hline Wa-CS & 0.001 & 0.308 & 0.009 & 0.090 & $\mathbf{0 . 0 1 7}$ & $\mathbf{0 . 0 0 0}$ \\
Wa-VI & $\mathbf{0 . 0 0 7}$ & $\mathbf{0 . 0 4 3}$ & 0.007 & 0.069 & 0.007 & 0.069 \\
CS-VI & 0.006 & 0.148 & $\mathbf{0 . 0 1 4}$ & $\mathbf{0 . 0 1 3}$ & $\mathbf{0 . 0 0 9}$ & $\mathbf{0 . 0 1 7}$ \\
\hline
\end{tabular}

1 